\title{
Incidence of prenatal alcohol exposure in Prince Edward Island: a population-based descriptive study
}

\author{
Janet Bryanton RN PhD, Joey Gareri MSc, Diane Boswall RN BN, Mary Jean McCarthy RN BN, \\ Bonnie Fraser RN BScN, Donna Walsh RN BN, Bridget Freeman MD BS, Gideon Koren MD, \\ Kathy Bigsby MD
}

\section{Abstract}

Background: Fetal alcohol spectrum disorder (FASD) is a leading preventable cause of neurodevelopmental disability in North America. The stigma associated with alcohol use and abuse during pregnancy makes it difficult to obtain information on prenatal alcohol use through self-reporting. We assessed the incidence of prenatal alcohol exposure in Prince Edward Island to facilitate future public health initiatives addressing FASD.

Methods: Prenatal alcohol exposure was examined via population-based collection of meconium and analysis of fatty acid ethyl esters (FAEEs). Fatty acid ethyl esters are nonoxidative metabolites of ethanol that are produced in the fetus. Meconium FAEE concentrations of $2.0 \mathrm{nmol} / \mathrm{g}$ or greater are indicative of frequent prenatal alcohol exposure during the last 2 trimesters of pregnancy. Samples were collected from 1307 neonates between Nov. 8, 2010, and Nov. 8, 2011, in hospitals in PEI, or from those born to mothers who resided in PEI but gave birth in Halifax, Nova Scotia. Samples were frozen and shipped for analysis. Fatty acid ethyl esters were analyzed by gas chromatography-mass spectrometry and quantified by means of deuterated internal standards.

Results: Of the 1307 samples collected, 1271 samples were successfully analyzed. Positive results for FAEEs were obtained in $3.1 \%$ ( $n=39$ ) of samples collected within the first 24 hours after birth.

Interpretation: Not all neonates exposed to heavy prenatal alcohol in utero will exhibit FASD; based on current estimates of predictive value for disease by exposure, our findings suggest that $1.3 \%$ of neonates born in PEI during this 1-year period will have FASD. In its application to an entire provincial birth cohort, this study successfully implemented a public health-centred approach for evaluating population-based risk of FASD, with implications for practice across Canada.

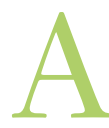

lcohol use in pregnancy is one of the leading preventable causes of developmental delays and birth defects in children in North America. ${ }^{1}$ Fetal alcohol spectrum disorder (FASD) occurs in about $40 \%$ of children exposed to frequent/intensive gestational alcohol use. ${ }^{2}$ Fetal alcohol spectrum disorder is a "spectrum" disorder, and thus exhibits a wide range of manifestations, extending from major malformations and severe developmental delays to more subtle impairments that can severely affect a person's ability to function successfully within society. ${ }^{3}$ The economic impact of this preventable disorder is large, with the adjusted annual costs of treatment for a person with FASD estimated at \$21 642. ${ }^{4}$ The annual cost of treatment for people with FASD aged 1 to 53 years across Canada is estimated at $\$ 5.3$ billion. ${ }^{4}$ Despite the substantial societal burden associated with FASD, prenatal screening for alcohol use is uncommon in routine clinical care. ${ }^{5}$ The established mechanisms of public health monitoring tend to be inadequate for reflecting the population-based risk levels for FASD. Traditional popu- lation monitoring is primarily dependent on maternal selfreporting, which - although effective in the detection of many health-related behaviours - is highly ineffective in identifying prenatal alcohol consumption because of the associated stigma.$^{6-8}$ Identifying children affected by in utero alcohol exposure is a major public health concern with regard to FASD. Only $10 \%$ of alcohol-affected children exhibit the pathognomonic craniofacial abnormalities required for a diagnosis of FASD in the absence of a confirmed history of prenatal ethanol exposure. ${ }^{2,9}$ This results in a large population of alcohol-affected people who are never

Competing interests: None declared.

This article has been peer reviewed.

Correspondence to: Joey Gareri, joey.gareri@sickkids.ca

CMAJ Open 2014.DOI:10.9778/cmajo.20140011 
identified and managed for this disorder, and who develop secondary disabilities that can lead to poverty, incarceration and early death.

Fatty acid ethyl esters (FAEEs) - metabolites of alcohol that can be detected in neonatal meconium - have emerged as biomarkers of prenatal alcohol consumption that are capable of identifying children at risk for FASD. ${ }^{6,10-13}$ Meconium comprises the neonate's first few bowel movements; its formation begins at about 12 weeks of gestation, when fetal swallowing of amniotic fluid is started. ${ }^{14,15}$ Xenobiotics and their metabolites are deposited into meconium via the biliary route or through fetal swallowing. ${ }^{14}$ Although the ontogeny of meconium suggests that its detection window may encompass the final 28 weeks of pregnancy; limited, but well-controlled clinical research suggests that the meconium detection window for certain compounds may be restricted to the final 4-8 weeks of pregnancy. ${ }^{16}$ The presence of elevated FAEE levels in meconium has been correlated with a myriad of adverse alcohol-related effects in animal studies. ${ }^{17,18}$ Human studies have also shown significant associations between meconium FAEE levels and adverse neonatal/pediatric outcomes, as well as a dose-response relation with maternal prenatal alcohol consumption. ${ }^{19-24}$ Unlike ethanol or other ethanol metabolites such as ethyl glucuronide (EtG), FAEEs do not cross the placenta, which means that meconium FAEEs represent alcohol metabolized within the fetus itself. ${ }^{25,26}$ Cumulative meconium FAEE concentrations of $2.0 \mathrm{nmol} / \mathrm{g}$ or greater are indicative of frequent prenatal ethanol exposure. ${ }^{6,27}$

An accurate population-based assessment to determine the incidence of prenatal alcohol exposure is important. It provides a sound basis for the allocation of resources to develop programs targeting the prevention of gestational alcohol use and early intervention after misuse during pregnancy. This study presents the use of meconium FAEE analysis as an anonymous universal screening tool, applied to an entire provincial birth cohort, to provide a population-based assessment of prenatal alcohol exposure.

We aimed to establish the provincial incidence of frequent prenatal alcohol exposure through the analysis of meconium samples anonymously collected from the entire population of live neonates born to women in PEI over a 1-year period.

\section{Methods}

\section{Sample collection}

The primary sites of sample collection were the maternity units of 2 general hospitals in PEI: the Queen Elizabeth Hospital and Prince County Hospital, which respectively reported about 930 births and 445 births during the study period. A secondary site of sample collection was the IWK Health Centre in Halifax, Nova Scotia. The IWK Health Centre is the primary referral hospital for mothers from PEI and newborns requiring tertiary care. About 25-30 babies are born to mothers from PEI or transferred to the centre annually; during the sample collection period, 34 neonates born to mothers from PEI were reported to be born at, or transferred to, the IWK Health Centre. Planned home births and births at the Moncton City Hospital were excluded from sample collection, because they are both rare. The institutional ethics review boards at all participating hospitals and the University of Prince Edward Island Research Ethics Board approved this study.

Meconium samples were anonymously collected by the nursing staff at each participating site, as part of the routine newborn protocol. The nursing staff were educated on sample collection and were provided with standard operating procedures by the project site coordinators. Each site coordinator was responsible for overseeing sample collection, freezing and shipping, and communicating with project leaders. The samples were collected for a consecutive 12-month period at each site from babies born from 8 am on Nov. 8, 2010, to 7:59 am on Nov. 8, 2011.

After they gave birth, the mothers were made aware of the study via posters on the maternity unit of each participating hospital. Pamphlets providing information on FASD and the rationale for the study were made available for mothers who requested more information. The nursing staff at each site were instructed to answer questions and reassure mothers of the universal and anonymous nature of the meconium collection. Because sample collection was anonymous, no consent was required; however, the parents could opt out of the study. To maintain anonymity, no identifying information was recorded on the collection containers. The collection containers contained only a randomly assigned code number and the time that had elapsed between birth and sample collection.

A minimum of $10 \mathrm{~mL}$ of meconium was collected from each live neonate. Ideally, the first meconium was collected; however, this was not always possible. Meconium was scraped out of soiled diapers and deposited into $15 \mathrm{~g}$ ointment jars (Amerisource Bergen). The specimen container was then placed into a biohazard bag and frozen at $-20^{\circ} \mathrm{C}$ within 12 hours of collection. Samples were couriered in a frozen condition on dry ice bimonthly to the Motherisk Drug Testing Laboratory at the Hospital for Sick Children in Toronto for analysis.

\section{FAEE analysis}

Samples received at the laboratory were kept frozen at $-80^{\circ} \mathrm{C}$ until analysis. Samples were analyzed according to a previously published method. ${ }^{28}$ Briefly, meconium was thawed at room temperature and homogenized, and about $500 \mathrm{mg}$ (minimum acceptable weight, $100 \mathrm{mg}$ ) was transferred to a $15 \mathrm{~mL}$ centrifuge tube (Fisher Scientific). Deuterated internal standards of ethyl palmitate, ethyl oleate, ethyl linoleate and ethyl stearate were added (Toronto Research Chemicals Inc.). Liquid-liquid extraction was done by adding a 5:2 heptane:acetone solution to each tube, which was vortexed for 1 minute. Samples were then centrifuged at $3500 \mathrm{rpm}(\sim 2500 \mathrm{~g})$ for 15 minutes at $4^{\circ} \mathrm{C}$. The heptane layer was then transferred to a $10 \mathrm{~mL}$ SPME vial (Supelco) and evaporated under nitrogen at $37^{\circ} \mathrm{C}$. Each sample was reconstituted in $1 \mathrm{~mL}$ of $0.1 \mathrm{M}$ phosphate buffer ( $\mathrm{pH}$ 7.6), capped and loaded onto the gas chromatography-mass spectrometry (GC-MS) for analysis. 
Headspace solid-phase microextraction of samples was done with the use of an AOC-5000 autosampler (Shimadzu Scientific Inc.) with a $65 \mu \mathrm{M}$ polydimethylsiloxane-divinylbenzene fibre (Sigma-Aldrich), followed by GC-MS analysis in the electron-ionization mode with the use of a Shimadzu QP2010 Plus (Shimadzu Scientific Inc.) with a FactorFour capillary column (Agilent). Chromatographic and mass spectrometry data were analyzed by means of the GCMSolutions software (Shimadzu Scientific Inc.). Concentrations of FAEE were determined against a validated 2-point calibration curve run, with each batch containing calibration standards comprising blank meconium spiked with all 4 FAEE species (Sigma-Aldrich) at a concentration of $400 \mathrm{ng}$, as well as negative (blank meconium) and positive controls (2 levels). FAEE results are reported as the molar sum of all the 4 FAEE species tested (ethyl palmitate, ethyl oleate, ethyl linoleate and ethyl stearate) divided by the weight of the specimen. The limit of detection for this method is $0.4 \mathrm{nmol} / \mathrm{g}$. Samples containing FAEE concentrations of $2.00 \mathrm{nmol} / \mathrm{g}$ or greater are considered positive for prenatal alcohol exposure. ${ }^{27}$

\section{Statistical analysis}

Descriptive and comparative statistical analyses were conducted with the use of SigmaStat software, version 3.1.1.0 2004 (SysStat Software Inc.) and Microsoft Excel 2007. All data were non-normally distributed, and significance was defined as $p<0.05$. Mann-Whitney rank sum analysis was used to compare the unpaired data.

\section{Results}

In total, 1307 meconium samples were collected at the 3 hospitals; this constitutes $93 \%$ of the estimated 1409 live births that occurred during the study period. The reasons for missed specimen collections were human error (e.g., forgotten or misplaced samples), intrapartum meconium evacuation and neonatal complications (e.g., imperforate anus and neonatal death). There were no records of mothers declining to provide a sample.

Of the 1307 collected samples, 1271 yielded results that could be used for analysis; the remaining 36 samples $(2.8 \%)$ were of insufficient quantity to conduct testing $(<100 \mathrm{mg})$, were determined to be stool samples (i.e., product of postnatal digestion) or could not be analyzed after extraction owing to the presence of constituents that interfered with chromatography.

Of the 1271 successfully analyzed samples, a total of 1091 meconium specimens exhibited negative results for quantifiable amounts of FAEEs. However, 124 specimens contained detectable FAEEs at concentrations that were lower than those associated with prenatal alcohol exposure, with a mean concentration of $0.79 \pm 0.04$ (standard error of the mean) $\mathrm{nmol} / \mathrm{g}$ and a range of $0.40-1.99 \mathrm{nmol} / \mathrm{g}$ (95\% confidence interval [C] 0.72 0.86). A total of 56 samples exhibited positive results for FAEEs, wherein the concentrations were higher than the clinical cut-off for prenatal alcohol exposure, with a mean concentration of $18.61 \pm 5.47$ (standard error of the mean) $\mathrm{nmol} / \mathrm{g}$ and a range of $2.06-199.88 \mathrm{nmol} / \mathrm{g}$ (95\% CI 7.65-29.57). Thirty-nine of the FAEE-positive samples were collected within 24 hours of birth. FAEE-positive meconium from samples collected after 24 hours carry a false-positive risk (about 20-30\%) due to endogenous ethanol production in the neonatal gut by postnatal bacterial colonization ${ }^{29}$; therefore the incidence of prenatal alcohol exposure in this population is presented as a range conservatively excluding the samples collected after 24 hours at the low end and including all analytically positive samples at the high end (Table 1).

The results of the meconium analysis suggest a minimum incidence range of $3.1 \%-4.4 \%$ for frequent prenatal alcohol exposure in this neonatal population.

\section{Interpretation}

Prince Edward Island is a province of Canada with a current population of 146000 and an estimated neonatal population of 1409 babies born during the study period. ${ }^{30}$ The urban/ rural population proportions for PEI are about 47\%/53\% compared with $81 \% / 19 \%$ for Canada. ${ }^{30,31}$ Most residents of PEI are European in origin, with about 3.25\% Aboriginal (First Nations, Inuit and Metis people), compared with the overall value of $5.59 \%$ for Canada. ${ }^{30,31}$ Reliable data on the incidence of frequent alcohol use during pregnancy in PEI have been lacking, and determining this incidence is critical in

\begin{tabular}{|c|c|c|c|}
\hline \multirow[b]{2}{*}{ Time of sample collection } & \multicolumn{2}{|c|}{ Negative result } & \multirow{2}{*}{$\begin{array}{c}\text { Positive result } \\
\text { FAEE level } \\
>2.00 \mathrm{nmol} / \mathrm{g}\end{array}$} \\
\hline & $\begin{array}{l}\text { Undetectable } \\
\text { FAEE level }\end{array}$ & $\begin{array}{c}\text { FAEE level } \\
0.40-2.00 \mathrm{nmol} / \mathrm{g}\end{array}$ & \\
\hline$\leq 24 \mathrm{~h}$ after birth & 962 & 95 & 39 \\
\hline 25-48 h after birth & 49 & 17 & 12 \\
\hline$>48 \mathrm{~h}$ after birth & 14 & 3 & 1 \\
\hline Unknown & 66 & 9 & 4 \\
\hline Total & 1091 & 124 & 56 \\
\hline
\end{tabular}


assessing the extent and potential impact of this problem. The PEI Reproductive Care Program Perinatal Database showed a rate of self-reported alcohol use at any time during pregnancy of less than $1 \%$ in $2011,{ }^{32}$ which is $3-4$ times lower than the estimate determined in our study by meconium analysis for frequent, late gestational drinking. The heavy stigma associated with alcohol consumption in pregnancy results in both underreporting by pregnant women and reluctance on the part of their health care providers to ask about prenatal alcohol use. ${ }^{5,78}$ A repeated pattern has been emerging through published studies that alcohol and uncontrolled substance abuse in pregnancy is underrecognized by traditional means of public health monitoring..$^{7,12,13,33,34}$ This contributes to the substantial lack of dedicated programs that provide supportive intervention for women and neonates at risk and perpetuates the misperception among general practitioners, pediatricians and obstetricians that prenatal alcohol exposure does not occur within their clinical population.

Our population-based study was anonymous, and no attempt was made to identify specific mother-infant pairs. This design is critical in ensuring that the true incidence of maternal alcohol consumption is measured. Previous studies have shown an approximate $20 \%$ decrease in overall maternal participation in nonanonymous neonatal screening programs and a substantial $90 \%$ decrease in the participation of women frequently consuming alcohol prenatally. ${ }^{12,35}$ Despite such challenges, nonanonymous voluntary participation in meconium screening for prenatal alcohol exposure has shown promising signs as a means to deliver supportive, advocative maternal intervention (i.e., interventions that provide social as well as clinical support and focus on maternal well-being) and early identification and treatment in potentially affected neonates. A pilot study, using a meconium screening model based on voluntary participation in a high-risk population, identified 1 FAEE-positive child in a population of 30 screened infants (3\%); both the mother and child benefited from enhanced follow-up care provided by the local public health unit, and the neonate showed substantial developmental delays that received immediate attention by 8 months of age. ${ }^{10}$ In comparison, the current group of 28 neonatal disorders that are screened for in Ontario affect only $0.11 \%$ of the neonatal population, with a false-positive result rate of $88 \% .^{36}$

By using the most conservative estimate of our study findings, a minimum of $3.1 \%$ of babies born to PEI mothers were considered to be exposed to frequent prenatal alcohol consumption beyond the first trimester of pregnancy, which placed these children at risk for FASD. About $40 \%$ of these exposed babies (1.3\% of babies born in this population) will have $\mathrm{FASD}{ }^{2}$ this translates to an estimated range of at least 16-22 children with FASD in PEI born during the study period. The incidence of positive results of meconium FAEEs in PEI is almost identical to that in Grey-Bruce, Ontario, which - similar to PEI — includes a city of about 30000 inhabitants surrounded by rural areas. ${ }^{6}$ The similarity in findings between the study conducted in Grey-Bruce and the current PEI study suggests the potential generalizability of these findings to similar populations in Canada.

\section{Limitations}

As with any laboratory test, there are limitations to the use of meconium FAEEs to determine prenatal alcohol exposure, such as the inability to detect alcohol consumption during the first and possibly the second trimesters of pregnancy and the inability to detect infrequent, but potentially fetotoxic, patterns of prenatal alcohol consumption. Despite these limitations, meconium FAEE analysis is currently the most validated neonatal biomarker for the evaluation of chronic prenatal alcohol exposure. Ethyl glucuronide is another emerging biomarker in meconium that has shown similar sensitivity and specificity as FAEEs; $;^{37,38}$ however, meconium EtG has not been shown to out-perform meconium FAEEs in terms of sensitivity and specificity, and has not yet been shown to have any predictive value for adverse neonatal/pediatric outcomes or dose-response correlation against maternal alcohol consumption, as has been established for FAEEs. ${ }^{17-24}$ Other laboratory methods, such as urine analysis of ethanol, exhibit very low sensitivity due to the rapid elimination of ethanol, resulting in a detection window of less than 24 hours. ${ }^{39}$ Urinary EtG has a longer urinary detection window $(\sim 90 \mathrm{~h})$ than ethanol, and its detection window is similar to that of most drugs that are abused, but is far shorter than the detection window of meconium. ${ }^{40}$ Analysis of maternal blood biomarkers such as carbohydrate-deficient transferrin, mean corpuscular volume, aspartate aminotransferase, alanine aminotransferase and gamma-glutamyl transferase have been shown to be ineffective in identifying excessive alcohol use in pregnancy and exhibit a comparatively limited detection window of about 3 weeks. ${ }^{7,41,42}$ Maternal hair analysis for FAEEs and EtG is a viable alternative to meconium FAEE analysis in terms of determining chronic alcohol use $;^{41,43,44}$ however, the more invasive nature of hair collection makes it a less suitable method for population-based studies aiming to establish incidence values and maximize participation.

The accurate threshold dose of maternal alcohol consumption that is required to produce a positive FAEE result in meconium is unknown, as controlled dose-response studies in pregnancy are not ethically feasible. However, clinical studies have shown that incidental/low-level alcohol consumption during the second and third trimester will not cause positive meconium FAEE results and that positive FAEE results suggest a level of alcohol consumption that involves more than 7 drinks per week and/or 3 standard drinks per drinking day., ${ }^{6,19,27}$ As these are the established parameters of the meconium FAEE test, the term "frequent prenatal alcohol exposure" used when discussing the findings of the present study refers to the levels of maternal alcohol use associated with meconium FAEE-positive findings. Although the meconium FAEE test is highly sensitive, and shows a fivefold higher probability of detecting frequent prenatal alcohol exposure compared with routinely used questionnaire-based methods, ${ }^{6,34}$ the false-negative rate of the FAEE test may be up to $20 \%,{ }^{19}$ suggesting that the $3.1 \%$ incidence rate proposed in this study is a decidedly conservative estimate of frequent prenatal alcohol exposure in this population.

Fetal alcohol spectrum disorder in most children remains undiagnosed because of the stigma associated with prenatal 
alcohol use, the lack of access to diagnostic services and the lack of targeted programs for clinical identification and intervention. Therefore, it is unlikely that surveys of diagnosed cases can provide regional or national estimates of this condition, which is among the most preventable causes of developmental morbidities in Canadian children. Moreover, it is likely that estimates provided by population-based studies with meconium FAEEs will remain the closest estimate of population morbidity by in-utero exposure to alcohol.

\section{Conclusion}

In its application to an entire provincial birth cohort, this study successfully implemented a public health-centred approach for evaluating population-based risk of prenatal alcohol exposure. Because it would be exceedingly difficult to replicate the design of this study to evaluate the entire Canadian neonatal population, these findings can serve as a guidepost to physicians in other Canadian provinces, alerting them to a likely hidden burden of FASD and maternal mental health issues in their own clinical population that may have gone undetected by traditional means of public health evaluation. The 1-year prenatal alcohol exposure incidence of 3.1\%4.4\% established in our study demonstrates that the province of PEI has a similar public health burden of prenatal alcohol exposure as has been estimated in other parts of Canada. Our findings further suggest that $1.3 \%$ of neonates born in PEI during the 1-year period of our study will have FASD.

Although the avoidance of alcohol consumption is the best option for a woman who is or might become pregnant, there are many complex factors that may influence why a pregnant woman may consume alcohol. The results of the present study provides a basis for a discussion of a multifaceted, integrated approach to addressing primary prevention, early identification and intervention for FASD in PEI and across Canada.

\section{References}

1. Dell CA, Roberts, G. Research update: alcohol use and pregnancy: an important Canadian public health and social issue. Ottawa $(\mathrm{ON})$ : Health Canada; 2005. Available: www.addictionresearchchair.ca/wp-content/uploads/Alcohol-Use -and-Pregnancy-An-Important-Canadian-Health-and-Social-Issue.pdf (accessed 2014 Apr. 2).

2. Abel EL. An update on incidence of FAS: FAS is not an equal opportunity birth defect. Neurotoxicol Teratol 1995;17:437-43.

3. Streissguth AP, Barr H, Kogan J, et al. Understanding the occurrence of secondary disabilities in clients with fetal alcohol syndrome (FAS) and fetal alcohol effects (FAE). Seattle (WA): University of Washington School of Medicine: Fetal Alcohol and Drug Unit, Department of Psychiatry and Behavioral Sciences; 1996.

4. Stade B, Ali A, Bennett D, et al. The burden of prenatal exposure to alcohol: revised measurement of cost. Can f Clin Pharmacol 2009;16:e91-102.

5. Sarkar M, Burnett M, Carrière S, et al.; Fetal Alcohol Spectrum Disorder Advisory Workgroup. Screening and recording of alcohol use among women of child-bearing age and pregnant women. Can 7 Clin Pharmacol 2009;16:e242-63.

6. Gareri J, Lynn H, Handley M, et al. Prevalence of fetal ethanol exposure in a regional population-based sample by meconium analysis of fatty acid ethyl esters. Ther Drug Monit 2008;30:239-45.

7. Stoler JM, Huntington KS, Peterson CM, et al. The prenatal detection of significant alcohol exposure with maternal blood markers. F Pediatr 1998;133:346-52.

8. Russell M, Martier SS, Sokol RJ, et al. Detecting risk drinking during pregnancy: a comparison of four screening questionnaires. Am 7 Public Health 1996; 86:1435-9.

9. Sampson PD, Streissguth AP, Bookstein FL, et al. Incidence of fetal alcohol syndrome and prevalence of alcohol-related neurodevelopmental disorder. Teratology 1997;56:317-26.

10. Zelner I, Shor S, Lynn H, et al. Clinical use of meconium fatty acid ethyl esters for identifying children at risk for alcohol-related disabilities: the first reported case. 7 Popul Ther Clin Pharmacol 2012;19:e26-31.
11. Hutson JR, Magri R, Gareri JN, et al. The incidence of prenatal alcohol exposure in Montevideo Uruguay as determined by meconium analysis. Ther Drug Monit 2010;32:311-7.

12. Goh YI, Hutson JR, Lum L, et al. Rates of fetal alcohol exposure among newborns in a high-risk obstetric unit. Alcohol 2010;44:629-34.

13. Pichini S, Marchei E, Vagnarelli F, et al. Assessment of prenatal exposure to ethanol by meconium analysis: results of an Italian multicenter study. Alcobol Clin Exp Res 2012;36:417-24.

14. Kwong TC, Ryan RM. Detection of intrauterine illicit drug exposure by newborn drug testing. National Academy of Clinical Biochemistry. Clin Chem $1997 ; 43: 235-42$.

15. Miller V, Holzel A. Growth and development of endodermal structures. In Davis J, Dobbing J, editors. Scientific foundations in paediatrics. Philadelphia: WB Saunders; 1974. 281-96.

16. Kacinko SL, Jones HE, Johnson RE, et al. Correlations of maternal buprenorphine dose, buprenorphine, and metabolite concentrations in meconium with neonatal outcomes. Clin Pharmacol Ther 2008;84:604-12

17. Brien JF, Chan D, Green CR, et al. Chronic prenatal ethanol exposure and increased concentration of fatty acid ethyl esters in meconium of term fetal Guinea pig. Ther Drug Monit 2006;28:345-50.

18. Zelner I, Kenna K, Brien JF, et al. Meconium fatty acid ethyl esters as biomarkers of late gestational ethanol exposure and indicator of ethanol-induced multiorgan injury in fetal sheep. PLoS ONE 2013;8:e59168.

19. Bearer CF, Jacobson JL, Jacobson SW, et al. Validation of a new biomarker of fetal exposure to alcohol. 7 Pediatr 2003;143:463-9.

20. Derauf C, Katz AR, Frank DA, et al. The prevalence of methamphetamine and other drug use during pregnancy in Hawaii. 7 Drug Issues 2003;33:1001-16.

21. Noland JS, Singer LT, Arendt RE, et al. Executive functioning in preschool-age children prenatally exposed to alcohol, cocaine, and marijuana. Alcobol Clin Exp Res 2003;27:647-56.

22. Noland JS, Singer LT, Short EJ, et al. Prenatal drug exposure and selective attention in preschoolers. Neurotoxicol Teratol 2005;27:429-38.

23. Peterson J, Kirchner HL, Xue W, et al. Fatty acid ethyl esters in meconium are associated with poorer neurodevelopmental outcomes to two years of age. 7 Pediatr 2008;152:788-92.

24. Jacobson SW, Bearer CF, Jacobson JL, et al. Meconium fatty acid ethyl esters (FAEEs) as predictors of severity of fetal alcohol spectrum disorder. Alcohol Clin Exp Res 2006;30(s1):175A.

25. Matlow JN, Lubetsky A, Aleksa K, et al. The transfer of ethyl glucuronide across the dually perfused human placenta. Placenta 2013;34:369-73.

26. Chan D, Knie B, Boskovic R, et al. Placental handling of fatty acid ethyl esters: perfusion and subcellular studies. 7 Pharmacol Exp Ther 2004;310:75-82.

27. Chan D, Bar-Oz B, Pellerin B, et al. Population baseline of meconium fatty acid ethyl esters among infants of nondrinking women in Jerusalem and Toronto. Ther Drug Monit 2003;25:271-8.

28. Hutson JR, Rao C, Fulga N, et al. An improved method for rapidly quantifying fatty acid ethyl esters in meconium suitable for prenatal alcohol screening. Alcobol 2011;45:193-9.

29. Zelner I, Hutson JR, Kapur BM, et al. False-positive meconium test results for fatty acid ethyl esters secondary to delayed sample collection. Alcohol Clin Exp Res 2012;36:1497-506.

30. Province of Prince Edward Island: Thirty-ninth annual statistical review. Charlottetown (PEI): PEI Statistics Bureau, Department of Finance, Energy, and Municipal Affairs; 2012. Available: www.gov.pe.ca/photos/original/fema_asr2012.pdf (accessed 2014 Jan.)

31. National Household Survey. Ottawa (ON): Statistics Canada; 2011 Available: www12.statcan.gc.ca/nhs-enm/index-eng.cfm (accessed 2014 Jan.).

32. Prince Edward Island Reproductive Care Program. Prince Edward Island Reproductive Care Program Perinatal Database Report 2011. Charlottetown (PEI): Department of Health and Wellness; 2013.

33. Forman R, Klein J, Barks J, et al. Prevalence of fetal exposure to cocaine in Toronto, 1990-1991. Clin Invest Med 1994;17:206-11.

34. Pichini S, Puig C, Zuccaro P, et al. Assessment of exposure to opiates and cocaine during pregnancy in a Mediterranean city: preliminary results of the "Meconium Project". Forensic Sci Int 2005;153:59-65.

35. Zelner I, Shor S, Lynn H, et al. Neonatal screening for prenatal alcohol exposure: assessment of voluntary maternal participation in an open meconium screening program. Alcobol 2012;46:269-76.

36. 2009 Statistics. Ottawa (ON): Newborn Screening Ontario, Children's Hospital of Eastern Ontario; 2009. Available: www.newbornscreening.on.ca/bins/content _page.asp?cid=7-272\&lang=1 (accessed 2014 Apr.).

37. Morini L, Groppi A, Marchei E, et al. Population baseline of meconium ethyl glucuronide and ethyl sulfate concentrations in newborns of nondrinking women in 2 Mediterranean cohorts. Ther Drug Monit 2010 Mar. 23 [Epub ahead of print].

38. Bakdash A, Burger P, Goecke TW, et al. Quantification of fatty acid ethyl esters (FAEE) and ethyl glucuronide (EtG) in meconium from newborns for detection of alcohol abuse in a maternal health evaluation study. Anal Bioanal Chem 2010;396:2469-77.

39. Jones AW. Evidence-based survey of the elimination rates of ethanol from blood with applications in forensic casework. Forensic Sci Int 2010;200:1-20.

40. Moller M, Gareri J, Koren G. A review of substance abuse monitoring in a social 
services context: a primer for child protection workers. Can 7 Clin Pharmacol 2010;17:e177-93.

41. Kharbouche H, Faouzi M, Sanchez N, et al. Diagnostic performance of ethyl glucuronide in hair for the investigation of alcohol drinking behavior: a comparison with traditional biomarkers. Int 7 Legal Med 2012;126:243-50.

42. Neumann T, Spies C. Use of biomarkers for alcohol use disorders in clinical practice. (2003). Addiction 2003;98(Suppl 2):81-91.

43. Pragst F, Balikova MA. State of the art in hair analysis for the detection of drug and alcohol abuse. Clin Chim Acta 2006;370:17-49.

44. Concheiro M, González-Colmenero E, Lendoiro E, et al. Alternative matrices for cocaine, heroin, and methadone in utero drug exposure detection. Ther Drug Monit 2013;35:502-9.

Affiliations: School of Nursing (Bryanton, McCarthy), University of Prince Edward Island, Charlottetown, PEI; Motherisk Program (Gareri, Koren), The Hospital for Sick Children, Toronto, Ont.; PEI Reproductive Care Program (Boswall); Queen Elizabeth Hospital (Fraser, Bigsby), Charlottetown, PEI; Prince County Hospital (Walsh, Freeman), Summerside, PEI; Leslie Dan Faculty of Pharmacy (Koren, Gareri), University of Toronto, Toronto, Ont.

Contributors: Janet Bryanton and Joey Gareri share primary authorship of this manuscript. Janet Bryanton and Kathy Bigsby are co-primary investigators and contributed to the conception, design and operation of the study. Mary Jean McCarthy, Bonnie Fraser, Donna Walsh, Diane Boswall, Bridget Freeman and Kathy Bigsby contributed to study conception and design. Bonnie Fraser and Donna Walsh supervised sample collection.
Janet Bryanton, Kathy Bigsby, Gideon Koren and Diane Boswall contributed to securing project funding from the Public Health Agency of Canada. Joey Gareri and Gideon Koren provided toxicological analysis of the samples. Joey Gareri provided analysis of meconium data. Janet Bryanton, Joey Gareri, Gideon Koren and Kathy Bigsby contributed to the interpretation of data. Joey Gareri and Janet Bryanton drafted the manuscript. All of the authors contributed substantially to the conception and design of the study, provided critical review of the manuscript, and gave final approval for publication.

Funding: This study was supported by grants from the Public Health Agency of Canada through The Canadian Association of Paediatric Health Centres. Gideon Koren is supported by the Ivey Chair in Molecular Toxicology, Department of Medicine, University of Western Ontario.

Acknowledgements: The authors acknowledge the contributions of Hara Kempton, Queen Elizabeth Hospital Site Coordinator; Margaret Barrett, Prince County Hospital Site Coordinator; Sharon Stone, IWK Site Coordinator; the nurses of the maternity units at the Queen Elizabeth Hospital and Prince County Hospital; the neonatal intensive care unit at the IWK Health Centre for their assistance with sample collection; and Chitra Rao, Motherisk Laboratory technician.

Supplemental information: For reviewer comments and the original submission of this manuscript, please see www.cmajopen.ca/content $/ 2 / 2$ /E121/suppl/DC1 